http://dx.doi.org/10.4314/ajid.v7i1.2

\title{
THE GROWING RESISTANCE OF KLEBSIELLA PNEUMONIAE; THE NEED TO EXPAND OUR ANTIBIOGRAM: CASE REPORT AND REVIEW OF THE LITERATURE
}

\author{
Musa A. Garbati ${ }^{1 *}$, Areedj I. Al Godhair ${ }^{2}$ \\ ${ }^{1}$ Section of Infectious Diseases, Department of Medicine, and ${ }^{2}$ Clinical Pharmacy Department, Pharmacy \\ Administration, King Fahad Medical City, 11525, Riyadh, Saudi Arabia. \\ *E-mail: musagarbati@yahoo.com
}

\begin{abstract}
Carbapenemases are being increasingly reported in Enterobacteriaceae including Klebsiella pneumoniae causing considerable increases in morbidity and mortality with limited therapeutic options. Issues related to difficulties associated with pathogen identification and infection control have been identified as major obstacles to the control of these multi-drug resistant organisms. Identification of this enzyme in organisms not previously found to harbor them has added to the already existing challenge in the control of this growing problem. The case of a 60 year-old Saudi lady with diabetes, hypertension, pituitary adenoma, hypothyroidism, and obstructive sleep apnea who was admitted in our intensive care unit following a cardiac arrest is hereby presented. During the course of her treatment she acquired various infections that led to her exposure to antimicrobials from almost all classes at various times; including bacteremia due to a pan-drug resistant Klebsiella pneumoniae and multi-drug resistant Acinetobacter baumannii. She was successfully treated with a combination of colistin and amikacin. This case highlights the resurgence of colistin in clinical practice and also calls for the need to expand our antibiogram to include antibiotics not conventionally reported, especially in areas where drug resistance is a growing problem. Improving susceptibility detection methods for Klebsiella pneumoniae and hand hygiene could prove effective in reducing nosocomial infections. Involvement of clinical pharmacists in antimicrobial stewardship could reduce the development of antimicrobial drug resistance.
\end{abstract}

Key words: Klebsiella pneumoniae, pan-drug resistance, infection, intensive care unit.

\section{Introduction}

The rising incidence of carbapenemase-producing Klebsiella pneumoniae has caused considerable challenge to the infectious diseases (ID) physician due to lack of therapeutic options. This rise has led to significant morbidity and mortality (Patel et al., 2008). Other infectious bacteria producing enzymes identical to the Klebsiella pneumoniae carbapenemases (KPCs) are also emerging rapidly as a cause of multidrug-resistant infections worldwide (Quale J, 2008). Carbapenemases other than KPC have also been associated with Klebsiella pneumoniae. These associations have been reported from many geographic zones in both metallo- (IMP, VIM and NDM) and non-metallo (KPC and OXA-48) enzyme producing isolates of K. pneumoniae (CDC, 2010). These isolates are capable of hydrolyzing a broad spectrum of $\beta$-lactam antibiotics including penicillins, cephalosporins, carbapenems and monobactam. Failure to identify individuals colonized with these organisms, difficulty with inoculum preparation, and problems intrinsic to automated systems are some of the reasons associated with this rise (Quale J, 2008). Data on healthcare-associated infections reported to the Centers for Diseases Control and Prevention (CDC) from 2007 indicated that $8 \%$ of all Klebsiella isolates were carbapenem resistant as compared to a previous report of $<1 \%$ in 2000 (Srinivasan and Patel, 2008) barely 7 years earlier. This enzyme has recently been isolated in Pseudomonas (Villegas et al., 2007) and Acinetobacter (Robledo et al., 2010), organisms outside the Enterobacteriacae family. The mechanisms by which these multi-drug resistant gram negatives spread despite effective infection control measures remains to be elucidated (Quale J 2008).

We describe the case of a 60 -year-old Saudi lady with diabetes and other multiple co-morbidities who developed recurrent infections with multiple organisms during a prolonged stay in the intensive care unit (ICU). Being the first report of infection with this organism from our institution, we have tried to explore the patient's journey to pan-drug resistance and also briefly reviewed the literature.

\section{Case report}

The patient is a 60-year-old Saudi female with type II diabetes mellitus, hypertension, pituitary adenoma, hypothyroidism, obesity and obstructive sleep apnea. She was in the ICU for one year. During this period she was connected to the ventilator that proved difficult to wean. Her hospital stay was complicated by anoxic brain damage which she sustained after cardiopulmonary arrest. She was previously admitted via the emergency room with productive cough, fever and shortness of breath that subsequently required intubation and ICU admission.

Subsequent examination revealed bilateral pneumonia. Arterial blood gases showed a $\mathrm{pH}$ of $7.20 ; \mathrm{PCO}_{2}: 80 \mathrm{mmHg}$; $\mathrm{PO}_{2}$ : 66 mmHg. White Blood Count was $12.4 \times 10^{9} / \mathrm{L}$, hemoglobin: $12.4 \mathrm{~g} / \mathrm{dl}$; and Platelet count: $203 \times 10^{9} / \mathrm{L}$. Chest radiography 
showed cardiomegaly with bilateral non-homogenous infiltrates. The admission diagnosis of community acquired pneumonia (CAP) with type II respiratory failure was made. The subject had a long ICU stay connected to a ventilator, had undergone several invasive procedures, and received immunosuppressive medications. Additionally, the patient was previously exposed to antibiotics belonging to nearly all classes (Penicillins, Carbapenems, Cephalosporins, Aminoglycosides, Glycopeptides, Glycylcyclines, Quinolones, and Polymyxins) due to infections caused by agents raging from sensitive Pseudomonas, E. coli to multi-drug resistant (MDR) Acinetobacter baumanii and extended-spectrum B-lactamases (ESBLs) Klebsiella pneumoniae before she acquired the infection under review.

About six months after her admission, she developed a new fever $\left(38.9^{\circ} \mathrm{C}\right.$,) with hypotension requiring inotropic support and high ventilator settings. Blood culture yielded pan-drug resistant Klebsiella pneumoniae (resistant to all tested antibiotics from all classes) and multi-drug resistant Pseudomonas aeruginosa (sensitive only to colistin and amikacin) at 12 hours of incubation in both aerobic and anaerobic vials. The Klebsiella pneumoniae isolate was not tested for colistin susceptibility but was confirmed as a carbapenemase producer by the modified Hodge test. No further characterization of the isolate was made. At the same time culture of aspirate from the trachea also yielded Pseudomonas aeruginosa that was sensitive only to colistin and amikacin. This patient was treated successfully with a combination of colistin and amikacin with complete resolution of both the pneumonia and bacteremia. Repeat blood culture 48 hours into her treatment with this combination was negative.

The patient was admitted in to a single room, under standard precautions applied, due to earlier infection with an ESBL organism. She remained isolated until her later transfer to a long term care facility for continuum of care.

\section{Discussion}

Infection with multi-drug resistant gram negative bacilli has been associated with significant morbidity and mortality (Patel et al., 2008). Bacterial isolates harboring carbapenemases are capable of hydrolyzing a broad spectrum of $\beta$-lactams including penicillins, cephalosporins, carbapenems and monobactam. Majority of these organisms belong to the ESCAPE (Enterococcus faecium, Staphylococcus aureus, Clostridium difficille, Acinetobacter baumannii, Pseudomonas aeruginosa, and Enterobacteriacae species) group of bacteria and are responsible for the majority of hospital- and community-acquired infections (Peterson, 2009). They tend to "escape" eradication by all currently available antimicrobial agents. This was highlighted in a recent review "bad bugs no drugs" from the Infectious Diseases Society of America (IDSA) (Boucher et al., 2009). This has become more worrisome especially at a time when no promising new antimicrobial agents are on the horizon (Talbot et al., 2006). A major risk factor for this rise is the increased use of carbapenems to treat extended-spectrum beta lactamase- (ESBL-) producing organisms with subsequent development of resistance to these agents (Yigit et al., 2001).

The rising number of immunocompromised patients has further led to the increased rate of infections with these highly resistant organisms (Chopra et al., 2008). Receipt of antibiotics containing the oxyimino group (cefuroxime, ceftriaxone, cefotaxime, ceftazidime, or aztreonam) is a well known risk factor for ESBL acquisition (Paterson et al., 2004). The optimal treatment of Carbapenemase-producing Klebsiella pneumoniae -producing organisms is yet unknown. With few new antimicrobials under development, clinicians have resorted to using older previously discarded antimicrobials, such as colistin and tigecycline to treat these infections (Urban et al., 2008). This approach has recently led to the emergence of organisms with resistance to antibiotics from all known classes, including the polymyxins (Zarkotou et al., 2010) with its attendant toxicities. tigecycline, which is bacteriostatic, is a glycylcycline with expanded activity against many enterobacteriaceae, including ESBLs and Klebsiella pneumoniae carbapenemases (KPCs). However, its low serum levels warrant caution when using it to treat bacteremic infections. The major concerns with colistin therapy are nephrotoxicity and neurotoxicity. Neurotoxicity might be difficult to ascertain especially in critically ill patients unable to report paraesthesia.

Carbapenemase-producing Klebsiella pneumoniae has been successfully treated with single or combination antimicrobial agents. A recent chart review by Neuner et al (Neuner et al., 2011) reported variable susceptibilities in vitro to tigecycline $(98 \%)$, colistin $(86 \%)$, amikacin $(45 \%)$ and gentamicin $(22 \%)$ among 60 patients with carbapenemase-producing Klebsiella pneumoniae bloodstream infections. Left with no other option clinicians have been forced to use alternative antibiotics such as tigecycline and the polymyxins (polymyxin B or colistin) to treat infections caused by carbapenemase resistant Enterobacteriacae as was in this case. The 10 by 20 initiative of the IDSA that aims to develop 10 new effective antibiotics by the year 2020 is a laudable program that is anticipated to provide effective alternatives (IDSA, 2010).

\section{Conclusion}

Klebsiella pneumoniae isolates resistant to all commonly used antimicrobial agents have been recognized as emerging infectious agents of clinical significance. The global spread of this agent is challenging for clinicians with very few therapeutic options left for countering these pathogens. Moreover, better methods are needed for isolating these pathogens and for screening individuals whom they colonize while we await the discovery of new active agents against resistant Gram-negative bacteria. The dearth of novel antibacterial drugs in the pipeline would mean that we must conserve the efficacy of existing antibacterial drugs as much as possible. While these needs are being addressed, it is also critical to have protocols in place for antimicrobial stewardship, enhance surveillance and institute effective infection control efforts to limit the spread of these pathogens. We share 
http://dx.doi.org/10.4314/ajid.v7i1.2

this case to highlight some troubling issues that soon may be relevant to increasing numbers of physicians and patients around the world.

\section{Informed consent}

Written informed consent was obtained from the patient for the publication of this case report.

\section{Disclosure}

The authors disclosed no conflict of interest in relation to this work.

\section{Acknowledgement}

The author is grateful to Dr. Jaffar Ali, Senior Editor, Research and Scientific Publications Center, King Fahad Medical Center for copyediting the manuscript.

\section{References}

1. Boucher, H. W., Talbot, G. H., Bradley, J. S., Edwards, J. E., Gilbert, D., Rice, L. B., Scheld, M., Spellberg, B., and Bartlett, J., (2009). Bad Bugs, No Drugs: No ESKAPE! An Update from the Infectious Diseases Society of America. Clin. Infect. Dis., 48: 1-12.

2. CDC (2010). Detection of Enterobacteriaceae isolates carrying metallo-beta-lactamase-United States, 2010. MMWR., 59: 750 .

3. Chopra, I., Schofield, C., Everett, M., O'Neill, A., Miller, K., Wilcox, M., Frère, J., Dawson, M., Czaplewski, L., Urleb, U., and Courvalin, P., (2008). Treatment of health-care associated infections caused by gram-negative bacteria: a consensus statement. Lancet Infect. Dis., 8: 133-139.

4. Infectious Diseases Society of America (2010). The 10 x '20 initiative: pursuing a global commitment to develop 10 new antibacterial drugs by 2020. Clin. Infect. Dis., 50: 1081-1083.

5. Neuner, E. A., Yeh, J. Y., Hall, G. S., Sekeres, J., Endimiani, A., Bonomo, R. A., Shrestha, N. K., Fraser, T. G., and van Duin, D., (2011). Treatment and outcomes in carbapenem-resistant Klebsiella pneumoniae bloodstream infections. Diagn. Microbiol. Infect. Dis., 69(4): 357-362.

6. Patel, G., Huprikar, S., Factor, S. H., Jenkins, S. G., and Calfee, D. P., (2008). Outcomes of carbapenem-resistant Klebsiella pneumoniae infection and the impact of antimicrobial and adjunctive therapies. Infect. Control Hosp. Epidemiol., 29(12): 1099-1106.

7. Paterson, D. L., Wen-Chien, K., Gottberg, A. V., Mohapatra, S., Casellas, J. M., Goossens, H., Mulazimoglu, L., Trenholme, G., Klugman, K. P., Bonomo, R. A., Rice, L. B., Wagener, M. M., McCormack, J. G., and Yu, V. L., (2004). International Prospective Study of Klebsiella pneumoniae Bacteremia: Implications of Extended-Spectrum $\beta$-Lactamase Production in Nosocomial Infections. Ann. Intern. Med., 140: 26-32.

8. Peterson, L. R. (2009). Bad Bugs, No Drugs: No ESCAPE Revisited. CID., 49(6): 992-993.

9. Quale, J. (2008). Global Spread of Carbapenemase-Producing Klebsiella pneumoniae. Microbe., 3(11): 516-520.

10. Robledo, I. E., Aquino, E. E., Sante, M. I., Santana, J. L., Otero, D. M., León, C. F. and Vázquez GJ (2010). Detection of KPC in Acinetobacter sp. in Puerto Rico. Antimicrob. Agents Chemother., 54: 1354-1357.

11. Srinivasan, A., and Patel, J. B. (2008). Klebsiella pneumoniae carbapenemase-producing organisms: an ounce of prevention really is worth a pound of cure. Infect. Control Hosp. Epidemiol., 29: 1107-1109.

12. Talbot, G. H., Bradley, J., Edwards, J. E. Jr, Gilbert, D., Scheld, M., and Bartlett, J. G. (2006). Bad bugs need drugs: an update on the development pipeline from the Antimicrobial Availability Task Force of the Infectious Diseases Society of America. CID., 42: 657-668.

13. Urban, C., Bradford, P. A., Tuckman, M., Maurer, S., Wehbeh, W., Grenner, L., Urban, R. C., Mariano, N., and Rahal, J. J. (2008). Carbapenem-resistant Escherichia coli harboring Klebsiella pneumoniae carbapenemase blactamases associated with long-term care facilities. Clin. Infect. Dis., 46: e127-130.

14. Villegas, M. V., Lolans, K., Correa, A., Kattan, J. N., Lopez, J. A., Quinn, J. P., and the Colombian Nosocomial Resistance Study Group (2007). First identification of Pseudomonas aeruginosa isolates producing a KPC-type carbapenem-hydrolyzing $\beta$-lactamase. Antimicrob. Agents Chemother., 51: 1553-1555.

15. Yigit, H., Queenman, A. M., Anderson, G. J., Domenech-Sanchez, A., Biddle, J. W., Steward, C. D., Alberti, S., Bush, K., and Tenover, F. C. (2001). Novel carbapenemhydrolyzing b-lactamase, KPC-1, from a carbapenemresistant strain of Klebsiella pneumoniae. Antimicrob. Agents Chemother., 45: 1151-1161.

16. Zarkotou, O., Pournaras, S., Voulgari, E., Chrysos, G., Prekates, A., Voutsinas, D., Themeli-Digalaki, K., and Tsakris, A. (2010). Risk Factors and Outcomes Associated with Acquisition of Colistin-Resistant KPC-Producing Klebsiella pneumoniae: a Matched Case-Control Study. J. Clin. Microbiol., 48(6): 2271-2274. 